\title{
Perfil Clínico-Epidemiológico e Sobrevida Global em Pacientes com Adenocarcinoma de Pâncreas em um Hospital de Referência em Oncologia
}

https://doi.org/10.32635/2176-9745.RBC.2021v67n1.967

\author{
Clinical Epidemiological Profile and Global Survival in Patients with Pancreatic Adenocarcinoma at a Reference Hospital in \\ Oncology \\ Perfil Clínico-Epidemiológico y Supervivencia General en Pacientes com Adenocarcinoma de Páncreas em un Hospital \\ de Referencia em Oncología
}

Wanessa Cristina Farias da Silva'; Ana Gabriela Silva de Lima ${ }^{2}$; Heverton Valentim Colaço da Silva ${ }^{3}$; Rozangela Amorim Santos ${ }^{4}$

\section{RESUMO}

Introduçáo: A neoplasia maligna de pâncreas configura uma das neoplasias de maior mortalidade em todo o mundo, quase sempre atrelada a um prognóstico sombrio, principalmente quando associada à disseminação linfática e para órgãos distantes. Objetivo: Avaliar a sobrevida global em pacientes com adenocarcinoma de pâncreas atendidos em um centro especializado em oncologia. Método: No período de janeiro de 2011 a dezembro de 2014, foram avaliados retrospectivamente 71 prontuários. Os dados foram analisados pelo software STATA versão 14, utilizando análise de Kaplan-Meier e de regressão de Cox. O intervalo de confiança utilizado foi de $95 \%$ e considerado significante $\mathrm{p}<0,05$. Foram preservados os princípios éticos e da confidencialidade. Resultados: Houve predomínio do sexo masculino, raça parda e com idade superior a 61 anos ao diagnóstico. Quanto às características clínicas, 87,8\% dos tumores estavam localizados em cabeça de pâncreas. A dor abdominal (92,7\%) foi o sintoma mais frequente, seguida de perda progressiva de peso (79,3\%) e icterícia (57,3\%). A taxa de sobrevida em três meses de acompanhamento foi de 48,4\%. Conclusáo: O estudo evidencia que o câncer de pâncreas tem uma repercussão extremamente negativa, visto que a maioria dos pacientes recebe o diagnóstico em estágios avançados da doença, dificultando a possibilidade de tratamento curativo.

Palavras-chave: Neoplasias pancreáticas/epidemiologia; Análise de Sobrevida; Diagnóstico Tardio.

\section{ABSTRACT}

Introduction: Pancreatic malignant neoplasia represents one of the highest mortality neoplasms worldwide, almost always associated to a dismal prognosis, especially when associated with lymphatic spread and to distant organs. Objective: To assess the global survival in patients with pancreatic adenocarcinoma treated at a specialized oncology center. Method: From January 2011 to December 2014, 71 medical records were retrospectively evaluated. The data were analyzed using the STATA software version 14 , using the Kaplan-Meier curve and the Cox regression. The confidence interval used was $95 \%(\mathrm{p}<0.05)$. Ethical and confidentiality principles have been secured. Results: There was predominance of males, mixed race and over 61 years of age at diagnosis. As for the clinical characteristics, $87.8 \%$ of the tumors were located in the head of the pancreas. Abdominal pain $(92.7 \%)$ was the most frequent symptom, followed by progressive weight loss $(79.3 \%)$ and jaundice $(57.3 \%)$. The three-month follow-up survival rate was $48.4 \%$. Conclusion: The study shows that pancreatic cancer has an extremely negative repercussion, since most patients are diagnosed in advanced stages of the disease, hindering the possibility of curative treatment. Key words: Pancreatic Neoplasms/epidemiology; Survival Analysis; Delayed Diagnosis.

\section{RESUMEN}

Introducción: La neoplasia maligna pancreática representa una de las neoplasias de mortalidad más altas del mundo, casi siempre vinculada a un pronóstico sombrío, especialmente cuando se asocia con diseminación linfática y órganos distantes. Objetivo: Evaluar la supervivencia global en pacientes con adenocarcinoma pancreático tratados en un centro de oncología especializado. Método: Desde enero de 2011 hasta diciembre de 2014, se evaluaron retrospectivamente 71 registros médicos. Los datos se analizaron utilizando el software STATA versión 14, utilizando la curva de Kaplan-Meier y la regresión de Cox. El intervalo de confianza utilizado fue del $95 \%(\mathrm{p}<0,05)$. Se han preservado los principios éticos y de confidencialidad. Resultados: Predominó el sexo masculino, la raza mixta y los mayores de 61 años en el momento del diagnóstico. En cuanto a las características clínicas, el $87,8 \%$ de los tumores se ubicaron en la cabeza del páncreas. El dolor abdominal (92,7\%) fue el síntoma más frecuente, seguido de pérdida progresiva de peso $(79,3 \%)$ e ictericia $(57,3 \%)$. La tasa de supervivencia de seguimiento a los tres meses fue del $48,4 \%$. Conclusión: El estudio muestra que el cáncer de páncreas tiene un impacto extremadamente negativo, ya que la mayoría de los pacientes reciben el diagnóstico en etapas avanzadas, obstaculizando la posibilidad de tratamiento curativo.

Palabras clave: Neoplasias Pancreáticas/epidemiología; Análisis de Supervivencia; Diagnóstico Tardío.

\footnotetext{
1,2,4 Hospital de Câncer de Pernambuco (HCP). Departamento de Ensino e Pesquisa (DEP). Santo Amaro. Recife (PE), Brasil. E-mails: wanessa farias@outlook.com; gabriela_27lima@hotmail.com; anjuamorim@yahoo.com. Orcid iD: https://orcid.org/0000-0002-8022-0091; Orcid iD: http://orcid.org/0000-0001-9041-4618; Orcid iD: https://orcid.org/0000-0002-5289-999X

${ }_{3}^{3}$ Universidade Federal de Pernambuco (UFPE). Departamento de Patologia. Cidade Universitária. Recife (PE), Brasil. E-mail: neto_pe6@live.com. Orcid iD: https:// orcid.org/0000-0001-7169-4140

Endereço para correspondência: Heverton Valentim Colaço da Silva. Departamento de Patologia da UFPE. Av. Prof. Moraes Rego, 1235 - Cidade Universitária. Recife (PE), Brasil. CEP 50670-420. E-mail: neto_pe6@live.com
} 


\section{INTRODUÇÃO}

O câncer de pâncreas é uma das neoplasias de maior mortalidade, ocupando o sétimo lugar em número de mortes relacionadas ao câncer ${ }^{1}$. Estima-se, para 2030, que o câncer de pâncreas seja a segunda causa de mortes por câncer nos Estados Unidos². Segundo o Instituto Nacional de Câncer José Alencar Gomes da Silva (INCA) ${ }^{3}$, este é responsável por cerca de $2 \%$ de todos os tipos de câncer diagnosticados e por $4 \%$ do total de mortes no Brasil.

Aproximadamente 95\% dos tumores malignos de pâncreas são do tipo histológico adenocarcinoma ${ }^{2}$. Sendo considerado uma das neoplasias malignas de pior prognóstico, apresenta taxa de sobrevida de cinco anos de apenas $5 \%{ }^{4}$.

Apesar de o câncer de pâncreas afetar ambos os sexos, a prevalência encontra-se aumentada no sexo masculino, cuja parcela menor dos casos ocorre em doentes com uma faixa etária dos 40 aos 50 anos, e o restante com idade acima dos 65 anos, sendo mais comum em indivíduos da raça negra ${ }^{5,6}$. Estudos populacionais sugerem que alguns casos de carcinoma pancreático podem ser de origem hereditária, reforçando a hipótese da predisposição genética ${ }^{7}$. Estima-se que, aproximadamente, $10 \%$ dos doentes com adenocarcinoma de pâncreas têm ou terão pelo menos um parente de primeiro ou de segundo grau acometido $^{3,4}$.

De difícil detecção, não possui rastreamento preconizado pelo $\mathrm{INCA}^{2,3}$. Seu prognóstico sombrio está associado à rápida disseminação no sistema linfático e órgáos distantes, bem como também à ausência de sintomas específicos da doença que possam conduzir ao diagnóstico precoce 5 . Esses fatores direcionam para uma previsão de que, em 2040, exista um aumento de sua incidência em nível mundial, com uma expectativa de 355.317 mil novos casos de câncer de pâncreas ${ }^{8}$.

Ao diagnóstico, apenas $20 \%$ dos pacientes podem ser submetidos a procedimentos cirúrgicos. A cirurgia é considerada a única terapia curativa existente até o momento e, mesmo assim, a taxa de sobrevida é, em média, de 12 meses $^{9,10}$, após esse procedimento.

Para os tumores irressecáveis e sem evidências de metástase a distância, é recomendada a associação de quimioterapia/radioterapia, visando ao controle local da doença da dor e à melhora na qualidade de vida, com relativo aumento da sobrevida média; entretanto, raros sáo os casos de pacientes com sobrevida em longo prazo ${ }^{11}$. Já nos pacientes que apresentam metástase a distância, é indicada quimioterapia paliativa com gencitabina. A sobrevida média para os pacientes com doença metastática é em torno de seis a nove meses ${ }^{12}$.
Portanto, o presente estudo tem como objetivo descrever as principais características epidemiológicas e clínicas relacionadas ao adenocarcinoma de pâncreas e estimar a sobrevida global dos pacientes atendidos no Hospital de Câncer de Pernambuco no período de 2011 a 2015.

\section{MÉTODO}

O estudo constou de uma abordagem retrospectiva, descritiva e quantitativa. Os dados foram provenientes do Registro do Hospitalar de Câncer (RHC) do Hospital de Câncer de Pernambuco e do Sistema de Informação sobre Mortalidade (SIM) do Estado de Pernambuco. Foram incluídos na pesquisa pacientes com diagnóstico de adenocarcinoma de pâncreas no período 1 de janeiro de 2011 a 31 de dezembro de 2015.

Foram critérios de inclusão deste estudo: idade igual ou acima de 18 anos, apresentar diagnóstico de adenocarcinoma de pâncreas e confirmaçáo do evento esperado (óbito). As variáveis analisadas foram: idade, sexo, etnia, histórico familiar, alcoolismo, tabagismo, comorbidades existentes: hipertensão arterial sistêmica (HAS), diabetes mellitus e obesidade, sintomas que motivaram a procura do atendimento hospitalar, data da biópsia, estadiamento, tratamento e a ocorrência do óbito. Foram excluídos da análise pacientes diagnosticados fora do período do estudo e que náo apresentaram biópsia de adenocarcinoma de pâncreas. Preencheram os critérios exigidos pelo estudo 71 pacientes. Foram excluídos da análise 22 .

Na descrição da população em estudo, foi apresentada a distribuição de frequências absolutas e percentuais. $\mathrm{Na}$ análise da mortalidade, foi aplicada a análise de sobrevida, sendo representada graficamente por curva de Kaplan-Meier e estimada a taxa de mortalidade em óbitos por cada 100 pacientes por mês, com intervalo de confiança de $95 \%$. A significância estatística adotada no estudo foi de 5\% ( $\mathrm{p}<0,05)$ e o software utilizado o STATA versão 14.

O estudo foi submetido ao Comitê de Ética em Pesquisa com seres humanos, por meio da Plataforma Brasil, sendo aprovado com número CAAE 84665418.2.0000.5205.

\section{RESULTADOS}

Dos 71 pacientes do estudo, 47 (66,19\%) tinham idade entre 35 a 64 anos. A média de idade ao diagnóstico foi de 61 anos. A maioria dos pacientes era do sexo masculino (n: $40 ; 56,3 \%$ ) e aproximadamente $60,56 \%$ dos pesquisados se declararam pardos. Quanto aos hábitos de vida, aproximadamente 34\% (n: 24) dos pacientes referiram ser tabagista e fazer uso de bebidas alcoólicas $(35,2 \%)$ (Tabela 1). 
Tabela 1. Caracterização da amostra composta por pacientes acometidos por adenocarcinoma de pâncreas, atendidos no Hospital de Câncer de Pernambuco, de janeiro de 2011 a dezembro de 2015

\begin{tabular}{lc}
\hline Fatores & Estatísticas \\
\hline Faixa etária & \\
35 a 50 anos & $16(22,5 \%)$ \\
De 51 a 65 anos & $31(43,7 \%)$ \\
Mais de 65 anos & $24(33,8 \%)$ \\
Sexo & \\
Feminino & $31(43,7)$ \\
Masculino & $40(56,3 \%)$ \\
Etnia & \\
Branca & $9(12,7 \%)$ \\
Preta & $19(26,8 \%)$ \\
Parda & $43(60,5 \%)$ \\
Hábitos de vida ${ }^{a}$ & \\
Não refere & $22(30,9 \%)$ \\
Tabagismo & $24(33,8 \%)$ \\
Etilismo & $25(35,2 \%)$ \\
Comorbidades ${ }^{a}$ & $19(1,4 \%)$ \\
Não apresenta comorbidades & $24(33,8 \%)$ \\
Hipertensão arterial sistêmica & $23(32,4 \%)$ \\
Diabetes mellitus & $23(32,4 \%)$ \\
Pancreatite & \\
Nãória familiar de neoplasia & \\
Sim & \\
\hline & \\
\hline
\end{tabular}

Legenda: ${ }^{\text {a }}$ Categorias náo excludentes.

Em relação à localização de maior frequência, 61 pacientes $(85,9 \%)$ possuíam tumores em cabeça de pâncreas. Entre os sintomas que motivaram a procura do atendimento hospitalar, os mais comuns foram dor abdominal (n: 64; 91,5\%) seguida de perda progressiva de peso (n: 46; 64,7\%) e icterícia (n: 41; 57,7\%). Os dados referentes às características do tumor estáo descritos na (Tabela 2).

As modalidades de tratamento do estudo incluíram cirurgia curativa em $17(23,9 \%)$ indivíduos. Nenhum desses pacientes foi submetido a modalidades de tratamento antes da cirurgia. Entre os pacientes submetidos à cirurgia curativa, $12(16,9 \%)$ também receberam quimioterapia adjuvante. Sete pacientes foram submetidos à cirurgia paliativa. A modalidade de escolha foi à cirurgia de derivaçáo biliar. Na população, apenas 32 pacientes foram submetidos ao tratamento quimioterápico com intuito paliativo ou curativo (Tabela 3 ).

A taxa de mortalidade foi de 11,8 óbitos por mês a cada 100 pacientes diagnosticados (IC 95\%: 9,4 a 14,9).
Tabela 2. Características do tumor dos pacientes acometidos por adenocarcinoma de pâncreas, atendidos no Hospital de Câncer de Pernambuco, de janeiro de 2011 a dezembro de 2015

\begin{tabular}{lc}
\hline Fatores & Estatísticas \\
\hline Local de acometimento & $10(14 \%)$ \\
Outros locais & $61(86 \%)$ \\
Cabeça de pâncreas & \\
Localização do tumora & \\
Local & $3(4,2 \%)$ \\
Localmente avançado & $9(12,7 \%)$ \\
Metastático & $59(83,1 \%)$ \\
Linfonodo acometido & \\
Não & $12(17 \%)$ \\
Sim & $59(83 \%)$ \\
Metástase & \\
Não & $12(17 \%)$ \\
Um local acometido & $49(69 \%)$ \\
Mais de um local acometido & $10(14 \%)$ \\
Estadiamento & \\
IA e IB & $9(12,6 \%)$ \\
IIA e IIB & $6(8,5 \%)$ \\
III & $6(8,5 \%)$ \\
IV & $50(70,4 \%)$ \\
\hline
\end{tabular}

Legenda: ${ }^{\text {a }}$ Categorias não excludentes.

Tabela 3. Características relacionadas ao tratamento dos pacientes acometidos por adenocarcinoma de pâncreas, atendidos no Hospital de Câncer de Pernambuco, de janeiro de 2011 a dezembro de 2015

\begin{tabular}{lc}
\hline Características & Número (\%) \\
\hline Tratamento & $31(43,6 \%)$ \\
Paliativo exclusivo & $23(32,3 \%)$ \\
Paliativo & $7(9,8 \%)$ \\
Cirurgia paliativa & $16(22,5 \%)$ \\
Quimioterapia paliativa exclusiva & $17(23,9 \%)$ \\
Cirúrgico curativo $^{a}$ & $12(16,9 \%)$ \\
Quimioterapia adjuvante & $1(1,4 \%)$ \\
Radioterapia adjuvante ${ }^{a}$ & \\
Tipo de cirurgia & $7(9,8 \%)$ \\
Duodenopancreatectomia & $10(14 \%)$ \\
Gastroduodenopancreatectomia & $7(9,8 \%)$ \\
Derivação exclusiva & \\
Protocolo quimioterápico & $20(28,1 \%)$ \\
Gemzar & $5(7 \%)$ \\
Outros & $7(7 \%)$ \\
Mais de um protocolo & \\
\hline
\end{tabular}

Legenda: ${ }^{\mathrm{a}}$ Procedimento com intuito curativo. 
O tempo médio de acompanhamento dos pacientes foi de 7,3 meses. A principal causa do óbito foi a sepse. A probabilidade de óbito dos pacientes com adenocarcinoma de pâncreas em seis meses após o diagnóstico foi de $62,9 \%$. Com base nos dados sobre o acompanhamento dos pacientes, evidenciou-se a evolução do percentual de óbito de 48,4\% a 75,2\% nos períodos de três e 12 meses, respectivamente (Tabela 4; Figura 1).

Tabela 4. Estatística dos óbitos e descritiva do acompanhamento

\begin{tabular}{lc}
\hline Características & Estatísticas \\
\hline Número de óbitos & 71 óbitos \\
Causa do óbito & \\
Sepse & $19(26,8 \%)$ \\
Insuficiência renal & $4(5,6 \%)$ \\
Insuficiência respiratória & $7(9,9 \%)$ \\
PCR & $5(7,0 \%)$ \\
Caquexia neoplásica & $8(11,3 \%)$ \\
Complicações pós-operatória & $7(9,9 \%)$ \\
Outras causas & $21(29,5 \%)$
\end{tabular}

Tempo de acompanhamento

Média (mínimo - máximo)

7,3 meses (3 dias $-5,4$ anos)

Taxa de mortalidade (IC 95\%)

Óbitos por mês a cada 100 diagnosticados

$11,8(9,4-14,9)$

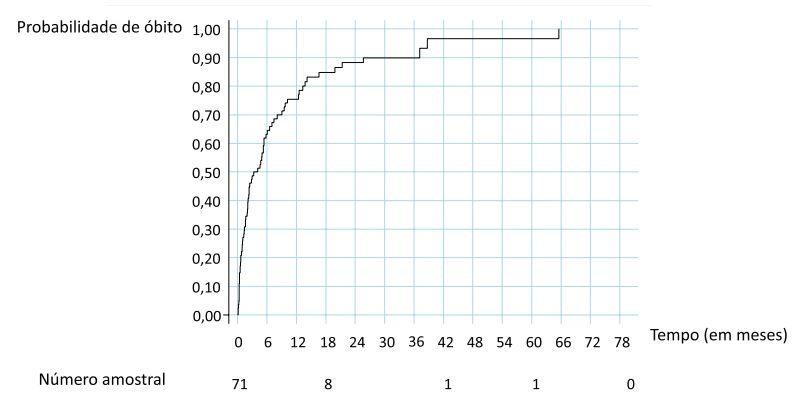

Figura 1. Probabilidade de sobrevida dos pacientes acometidos por adenocarcinoma de pâncreas, atendidos no Hospital de Câncer de Pernambuco, de janeiro de 2011 a dezembro de 2015

\section{DISCUSSÃO}

$\mathrm{Na}$ presente pesquisa, a análise descritiva encontrou maior prevalência de indivíduos do sexo masculino, o que pode ser justificado pela maior presença do tabagismo nesse sexo, o que é um importante fator de risco para o desenvolvimento de neoplasias ${ }^{10}$. Aproximadamente 20\% dos cânceres de pâncreas estão associados ao tabagismo, e indivíduos tabagistas apresentam risco aumentado para o desenvolvimento de mutaçôes genéticas 5 .

Foi encontrado no estudo um predomínio de pacientes acometidos na sexta década de vida, tais padróes são compatíveis com dados recentes do INCA $^{3}$. A média de idade evidenciada corrobora o estudo de Kongkam et al. ${ }^{13}$, que avaliou 100 pacientes com diagnóstico de adenocarcinoma de pâncreas e com idade média ao diagnóstico de 62,7 anos.

Com relação à raça, observou-se que o estudo diverge da literatura, uma vez que esta aponta um maior acometimento do câncer pancreático em indivíduos negros $^{3,4}$. A maioria dos pacientes do estudo declarou-se parda. Uma hipótese que tenta justificar essa divergência é o fato de a miscigenação racial ser tão presente no Brasil.

$\mathrm{Na}$ casuística apresentada, 26 pacientes $(32,3 \%)$ relataram diabetes mellitus. A literatura aponta que a incidência de diabetes está aumentada em pacientes com câncer pancreático, mas a relação entre ela e o câncer é controversa ${ }^{8}$. Para Andersen et al. ${ }^{14}$, o diabetes mellitus pode também ser uma manifestação precoce do câncer de pâncreas em vez de apenas um fator de risco. Para Liao et al. ${ }^{15}$, diabéticos diagnosticados com menos de dois anos possuem elevado risco para o desenvolvimento de câncer de pâncreas. Entretanto, pacientes com diagnóstico acima desse intervalo de tempo náo apresentaram diferença significante.

Sobre o consumo de álcool e o aumento do risco de câncer de pâncreas, os dados encontrados na literatura não são completamente estabelecidos, pois os atuais estudos apresentam limitaçóes em suas correlaçóes ${ }^{16}$. Contudo, a literatura associa o alcoolismo ao desenvolvimento de injurias no pâncreas e a pancreatite é considerada um fator de risco para o desenvolvimento de neoplasias pancreáticas $^{16,17}$. Na população analisada, a maioria dos indivíduos referiu fazer uso de bebidas alcoólicas.

Deve-se considerar que indivíduos que fazem uso abusivo de álcool geralmente são tabagistas e o tabaco é um fator de risco associado ao desenvolvimento de câncer de pâncreas ${ }^{16}$.

Neste estudo, a dor abdominal, a perda progressiva de peso e a icterícia foram as queixas mais comuns relatadas e, muitas vezes, costumam ser os primeiros sinais de alerta para a investigação e diagnóstico. O tipo de tumor e a sua localização costumam definir a sintomatologia inicial, de forma que, aproximadamente, $70 \%$ dos adenocarcinomas de pâncreas localizam-se na região cefálica do órgão e, em razão da sua proximidade com as vias biliares, podem apresentar sintomas ainda quando potencialmente curáveis $^{18,19}$. Os tumores da cauda e corpo são habitualmente assintomáticos em estágios mais avançados e apresentam pior prognóstico ${ }^{20,21}$. Quanto mais avançados os tumores, 
maiores são as sintomatologias por eles produzidas que não se restringem às anteriormente citadas.

O resultado dos sintomas descritos nesta análise é comparável com estudo realizado com 50 pacientes com adenocarcinoma de pâncreas na população asiática, sendo constatada a presença de dor abdominal em $72 \%$ dos pacientes estudados ${ }^{22}$. Em outro estudo realizado na Inglaterra com 119 pacientes com câncer de pâncreas com idade a partir de 40 anos, puderam-se observar, entre os sintomas mais encontrados, a presença de icterícia $(51 \%)$ e a perca de peso $(55 \%)$, corroborando os achados encontrados no respectivo estudo ${ }^{23}$. Os achados também condizem com o estudo de Reddy et al. ${ }^{24}$, em que a dor abdominal foi o sintoma mais comum, acometendo $84 \%$ dos indivíduos estudados ${ }^{22}$. Portanto, com base nos resultados desses estudos, em indivíduos com idade a partir dos 40 anos que apresentam queixa de dor abdominal, perda progressiva de peso, sinais de icterícia, fatores de risco hereditários e presença de massa sólida pancreática, deve-se proceder à investigaçáo por suspeita de câncer pancreático.

O estadiamento é um passo importante no gerenciamento das condutas a serem seguidas em relação ao tratamento proposto ${ }^{23}$. Desse modo, tumores diagnosticados em estágios iniciais requerem tratamentos menos agressivos e apresentam melhores desfechos ${ }^{25}$. Atualmente, a ressecção cirúrgica continua sendo a única opção curativa para o câncer de pâncreas, porém, em virtude da sua apresentaçấo tardia, cerca $20 \%$ dos pacientes são candidatos à realização do procedimento cirúrgico ${ }^{11}$. Esses dados corroboram os resultados do presente estudo, uma vez que apenas $23,9 \%$ dos pacientes realizaram procedimento cirúrgico com intuito curativo, sendo que $83 \%$ do total de pacientes já apresentavam doença metastática.

O estádio da doença, no momento do diagnóstico, tem sido considerado por diversos autores um fator determinante na sobrevida dos paciente ${ }^{26}$. O diagnóstico tardio pode ser uma das razóes para uma maior probabilidade de doença localmente avançada ou metastática $^{10}$. O acesso restrito à saúde e a baixa escolaridade sáo fatores que podem influenciar no diagnóstico da doença, fazendo com que a descoberta tardia da doença passe de um estágio potencialmente curável, para cuidados paliativos ${ }^{27}$.

Tumores diagnosticados em estágio avançado e metastático apresentam como modalidade de tratamento a quimioterapia e/ou radioterapia ${ }^{11,28}$. Mas, por causa da sua alta toxicidade e da incidência de efeitos adversos, são indicadas apenas para pacientes que apresentam condiçôes clínicas e bom desempenho ${ }^{28}$. Neste estudo, evidenciou-se que apenas um paciente $(1,4 \%)$ foi indicado à modalidade de tratamento com quimioterapia associada à radioterapia.
Ao analisar a sobrevida global após seguimento de três meses, 48,4\% evoluíram à óbito e, em um ano do diagnóstico, essa probabilidade evoluiu para $75,2 \%$. Em um comparativo com este estudo, em uma coorte realizada por Espindola et al. ${ }^{29}$, que avaliaram a sobrevida em pacientes com câncer de pâncreas em um período de dois anos, após um seguimento de cinco meses, a sobrevida foi de $47,64 \%$ e, em um ano, de $87 \%$. A taxa de sobrevida encontrada em ambos os estudos pode ser utilizada para estimar a eficiência do sistema de saúde, que depende da qualidade do cuidado prestado e da acessibilidade ao sistema de saúde.

O respectivo estudo mostra que, para a evolução do óbito, a causa mais frequente foi a sepse, seguida de caquexia neoplásica. É importante lembrar que o tratamento com quimioterapia antineoplásica, uso de corticoides, radioterapia e/ou cirurgia predispóe à imunossupressão $0^{30}$. Além disso, a recorrência de internamentos expóe o paciente a artefatos invasivos que aumentam os riscos infecciosos ${ }^{31}$.

Algumas limitaçóes do estudo precisam ser reconhecidas. Além das questôes inerentes ao não preenchimento correto dos prontuários por parte dos profissionais de saúde, a falta de uma avaliação funcional da saúde do paciente prejudicou a análise de algumas variáveis. Até o momento, este é um dos primeiros estudos a avaliar o perfil epidemiológico e clínico e o tempo de sobrevida global em pacientes diagnosticados com adenocarcinoma de pâncreas em um hospital de referência em oncologia na Regiáo Nordeste do Brasil, e deve ser útil no direcionamento do desenvolvimento de políticas de saúde para essa populaçáo.

\section{CONCLUSÃO}

Os resultados mostraram que as características clínico-epidemiológicas mais predominantes foram: tabagismo, etilismo, indivíduos da cor parda, diabetes mellitus, dor abdominal, perda progressiva de peso e icterícia. Grande parte da população analisada foi diagnosticada em estádios avançados, o que dificultou a possibilidade de tratamento curativo. $\mathrm{O}$ câncer de pâncreas continua sendo uma doença desafiadora para diagnosticar e tratar. Essa realidade evidencia, portanto, a necessidade de novos estudos na área, além de novas políticas públicas que promovam o diagnóstico precoce dessa neoplasia, que parecem ser as medidas mais importantes para melhorar as taxas de cura dessa enfermidade.

\section{CONTRIBUIÇÕES}

Todos os autores contribuíram na concepção e/ ou no planejamento do estudo; na obtenção, análise e 
interpretação dos dados; assim como na redação e revisão crítica; e aprovaram a versão final a ser publicada.

\section{DECLARAÇÃO DE CONFLITO DE INTERESSES}

Nada a declarar.

\section{FONTES DE FINANCIAMENTO}

Não há.

\section{REFERÊNCIAS}

1. Bray F, Ferlay J, Soerjomataram I, et al. Global cancer statistics 2018: GLOBOCAN estimates of incidence and mortality worldwide for 36 cancers in 185 countries. CA. Cancer J. Clin. 2018;68(6):394-424. doi: https:// doi.org/10.3322/caac.21492

2. Rahib L, Smith BD, Aizenberg R, et al. Projecting cancer incidence and deaths to 2030: the unexpected burden of thyroid, liver, and pancreas cancers in the United States. Cancer Res. 2014;74(11):2913-21. doi: https://doi. org/10.1158/0008-5472.CAN-14-0155

3. Instituto Nacional de Câncer José Alencar Gomes da Silva [Internet]. Rio de Janeiro: INCA; [data desconhecida]. Tipos de câncer: câncer de pâncreas; [modificado 2020 abr 4; acesso 2020 abr 4]. Disponível em: https://www. inca.gov.br/tipos-de-cancer/cancer-de-pancreas

4. Jomrich G, Gruber ES, Winkler D, et al. Systemic Immune-Inflammation Index (SII) predicts poor survival in pancreatic cancer patients undergoing resection. J GastrointestSurg. 2020;24(3):610-18. doi: https://doi. org/10.1007/s11605-019-04187-z

5. Bontempo L, Jácome GC, Bitencourt EL. Perfil epidemiológico do câncer de pâncreas na regiáo Norte do Brasil no período de 2010 a 2018. Rev Patol Tocantins. 2019;6(2):20-5. doi: https://doi.org/10.20873/uft.2446$6492.2019 \mathrm{v} 6 \mathrm{n} 2 \mathrm{p} 20$

6. Permuth JB, Clark Daly A, Jeong D, et al. Racial and ethnic disparities in a state- wide registry of patients with pancreatic cancer and an exploratory investigation of cancer cachexia as a contributor to observed inequities. Cancer Med. 2019;8(6):3314-24. doi: https://doi. org/10.1002/cam 4.2180

7. Malhotra L, Ahn DH, Bloomston M. The pathogenesis, diagnosis, and management of pancreatic cancer. J Gastrointest Dig Syst. 2015;5(2):1-11. doi: https://doi. org/10.4172/2161-069X.1000278

8. Rawla P, Sunkara T, Gaduputi V. Epidemiology of pancreatic cancer: global trends, etiology and risk factors. World J Oncol. 2019;10(1):10-27. doi: https://doi. org/10.14740/wjon1166

9. Usón Junior PLS, França MS, Rodrigues HV, et al. Maior sobrevida global em pacientes com câncer pancreático metastático: o impacto de onde e como o tratamento é realizado. Einstein. São Paulo. 2015;13(3):347-351. doi: https://doi.org/10.1590/S1679-45082015AO3303

10. Bittencourt CP, Abreu MC, Souza TF, et al. Tabagismo e sua relação com o desenvolvimento de câncer. Rev Cient ITPAC [Internet]. 2017 [acesso 2020 ago 12];10(1):1318. Disponível em: https://assets.unitpac.com.br/ arquivos/revista/2017-1/Artigo_2.pdf

11. Kamisawa T, Wood LD, Itoi T, et al. Pancreatic cancer. Lancet. 2016;388(10039):73-85. doi: https://doi. org/10.1016/S0140-6736(16)00141-0

12. McGuigan A, Kelly P, Turkington RC, et al. Pancreatic cancer: a review of clinical diagnosis, epidemiology, treatment and outcomes. World J Gastroenterol. 2018;24(43):4846-61. doi: https://doi.org/10.3748/ wjg.v24.i43.4846

13. Kongkam P, Benjasupattananun P, Taytawat $P$, et al. Pancreatic cancer in an Asian population. Endosc Ultrasound. 2015;4(1):56-62. doi: https://doi. org/10.4103/2303-9027.151361

14. Andersen DK, Korc M, Petersen GM, et al. Diabetes, pancreatogenic diabetes, and pancreatic cancer. Diabetes. 2017;66(5):1103-10. doi: https://doi.org/10.2337/ db16-1477

15. Liao KF, Lai SW, Li CI, et al. Diabetes mellitus correlates with increased risk of pancreatic cancer: a populationbased cohort study in Taiwan. J Gastroenterol Hepatol. 2012;27(4):709-13. doi: https://doi.org/10.1111/ j.1440-1746.2011.06938.x

16. Korc M, Jeon CY, Edderkaoui M, et al. Tobacco and alcohol as risk factors for pancreatic cancer. Best Pract Res Clin Gastroenterol. 2017;31(5):529-36. doi: https:// doi.org/10.1016/j.bpg.2017.09.001

17. Jayasekara H, English DR, Hodge AM, et al. Lifetime alcohol intake and pancreatic cancer incidence and survival: findings from the Melbourne Collaborative Cohort Study. Cancer Causes Control. 2019;30(4):323-31. doi: https://doi.org/10.1007/ s10552-019-01146-6

18. Walling A, Freelove R. Pancreatitis and pancreatic cancer. Prim Care. 2017;44(4):609-20. doi: https://doi. org/10.1016/j.pop.2017.07.004

19. Song TJ, Seo DW, Lakhtakia S, et al. Initial experience of EUS-guided radiofrequency ablation of unresectable pancreatic cancer. Gastrointest Endosc. 2016;83(2):4403. doi: https://doi.org/10.1016/j.gie.2015.08.048

20. Dreyer SB, Jamieson NB, Upstill-Goddard R, et al. Defining the molecular pathology of pancreatic body and tail adenocarcinoma. Br J Surg. 2018;105(2):e183-e191. doi: https://doi.org/10.1002/bjs.10772

21. van Erning FN, Mackay TM, van der Geest LGM, et al. Association of the location of pancreatic ductal adenocarcinoma (head, body, tail) with tumor stage, treatment, and survival: a population-based analysis. 
Acta Oncol. 2018;57(12):1655-62. doi: https://doi.org /10.1080/0284186X.2018.1518593

22. Kanno A, Masamune A, Hanada K, et al. Multicenter study of early pancreatic cancer in Japan. Pancreatology. 2018;18(1):61-7. doi: https://doi.org/10.1016/j. pan.2017.11.007

23. Walter FM, Mills K, Mendonça SC, et al. Symptoms and patient factors associated with diagnostic intervals for pancreatic cancer (SYMPTOM pancreatic study): a prospective cohort study. Lancet Gastroenterol Hepatol. 2016;1(4):298-306. doi: https://doi.org/10.1016/ S2468-1253(16)30079-6

24. Reddy S, Cameron JL, Scudiere J, et al. Surgical Management of Solid-Pseudopapillary Neoplasms of the Pancreas (Franz or Hamoudi Tumors): a large singleinstitutional series. J Am Coll Surg. 2009;208(5):950-7. doi: https://doi.org/10.1016/j.jamcollsurg.2009.01.044

25. Adamska A, Domenichini A, Falasca M. Pancreatic ductal adenocarcinoma: current and evolving therapies. Int J Mol Sci. 2017;18(7):1338. doi: https://doi.org/10.3390/ ijms 18071338

26. Zhang Q, Zeng L, Chen Y, et al. Pancreatic cancer epidemiology, detection, and management. Gastroenterol Res Pract. 2016;2016:8962321. doi: https://doi. org/10.1155/2016/8962321

27. Visentin A, Mantovani MF, Kalinke LP, et al. Palliative therapy in adults with cancer: a cross-sectional study. Rev Bras Enferm. 2018;71(2):252-8. doi: https://doi. org/10.1590/0034-7167-2016-0563

28. Kasuga A, Hamamoto Y, Takeuchi A, et al. Positive relationship between subsequent chemotherapy and overall survival in pancreatic cancer: meta-analysis of postprogression survival for first-line chemotherapy. Cancer Chemother Pharmacol. 2017;79(3):595-602. doi: https://doi.org/10.1007/s00280-017-3263-3

29. Espindola LMD, Mota A, Gomes EAP, et al. Sobrevida em dois anos de pacientes acometidos por câncer de pâncreas e os fatores associados. ACM Arq Catarin Med [Internet]. 2013 [acesso 2020 set 14];42(2)62-9. Disponível em: http://www.acm.org.br/acm/revista/pdf/ artigos/1230.pdf

30. Togashi Y, Shitara K, Nishikawa H. Regulatory T cells in cancer immunosuppression - implications for anticancer therapy. Nat Rev Clin Oncol. 2019;16(16):356-71. doi: https://doi.org/10.1038/s41571-019-0175-7

31. Almeida RVS, Pacheco-JR AM, Silva RA, et al. Angiolymphatic invasion as a prognostic fator in resected N0 pancreatic adenocarcinoma. ABCD Arq Bras Cir Dig. 2017;30(1):42-46. doi: https://doi.org/10.1590/01026720201700010012 